\title{
Tecnura
}

\section{Conciliación de inventarios top-down y bottom-up de emisiones de fuentes móviles en Bogotá, Colombia}

\section{Conciliation of top-down and bottom-up inventories to estimate mobile source emissions in Bogota, Colombia}

\author{
Luis Gabriel Carmona Aparicioํㅜㄹ Mauricio Alexander Rincón Pérez², \\ Andrés Mauricio Castillo Robles ${ }^{3}$, Boris René Galvis Remolina ${ }^{4}$, Hugo Enrique Sáenz Pulido ${ }^{5}$, \\ Rodrigo Alberto Manrique Forero ${ }^{6}$, Jorge Eduardo Pachón Quinche ${ }^{7}$
}

Fecha de recepción: 5 de mayo de 2015

Fecha de aceptación: 15 de mayo de 2016

Cómo citar: Carmona Aparicio, L. G., Rincón Pérez, M. A., Castillo Robles, A. M., Galvis Remolina, B. R., Sáenz Pulido, H. E., Manrique Forero, R. A., \& Pachón Quinche, J. E. (2016). Conciliación de inventarios top-down y bottom-up de emisiones de fuentes móviles en Bogotá, Colombia. Revista Tecnura, 20(49), 59-74. doi: 10.14483/ udistrital.jour.tecnura.2016.3.a04

\section{RESUMEN}

Objetivo: Este estudio presenta la conciliación del inventario de emisiones de fuentes móviles en Bogotá para el año 2013 aplicando las metodologías top-down y bottom-up.

Método: Una comparación de las emisiones de las diferentes categorías vehiculares fue realizada, así como la distribución geográfica y temporal de las mismas, mediante la creación de un código de procesamiento en JAVA. El proceso empleó la mayor cantidad de información disponible por las autoridades del Distrito Capital y estudios previos. La innovación particular del presente estudio consiste en la obtención de los flujos vehiculares de vías secundarias a partir de un algoritmo que considera geometría de la vía, interacción entre vías arteriales y secundarias, y restricciones legales de movilidad.

Resultados: Los resultados muestran la estimación de emisiones para los contaminantes $\mathrm{CO}, \mathrm{CO}_{2^{\prime}}$ $\mathrm{COV}, \mathrm{NO}_{x^{\prime}} \mathrm{SO}_{2}$ y $\mathrm{PM}$, además de su comparación con otros estudios realizados en la ciudad. Los valores obtenidos pueden ser catalogados como referentes mínimos y máximos de las emisiones emitidas para 2013, dada la incertidumbre de los datos.

Conclusiones: El proyecto proporciona una herramienta muy útil como insumo para las autoridades

Ingeniero Ambiental y Sanitario, magíster en Eficiencia Energética y Ecología Industrial. Docente e investigador en la Universidad Piloto de Colombia y en el Institute FORWARD - For Worldwide Alternative Research and Development. Bogotá, Colombia. Contacto: lugacapa@ gmail.com

Ingeniero Químico, magíster en Ingeniería Ambiental. Profesional de modelación de calidad del aire en la Secretaría Distrital de Ambiente de Bogotá. Bogotá, Colombia. Contacto: alexrinconp@gmail.com

Ingeniero de Sistemas, doctor en Ingeniería de Sistemas y Computación. Investigador en la Universidad Nacional de Colombia. Bogotá, Colombia. Contacto: amcastillor@unal.edu.co

4 Ingeniero Químico, magister en Ingeniería Ambiental, doctor en Ingeniería Ambiental. Docente de la Universidad de La Salle, investigador Centro Lasallista de Investigación y Modelación Ambiental CLIMA. Bogotá, Colombia. Contacto: bgalvis@unisalle.edu.co

5 Ingeniero Mecánico. Profesional Plan Decenal de Descontaminación del Aire de Bogotá, Secretaría Distrital de Ambiente. Bogotá, Colombia. Contacto: hugo.saenz@ambientebogota.gov.co

6 Ingeniero en Recursos Hídricos y Gestión Ambiental. Docente Universidad Autónoma de Colombia. Bogotá, Colombia. Contacto: rodrigo1358@icloud.com

Ingeniero Químico, magister en Ingeniería Ambiental, doctor en Ingeniería Ambiental. Docente de la Universidad de La Salle, investigador Centro Lasallista de Investigación y Modelación Ambiental CLIMA. Bogotá, Colombia. Contacto: jpachon@unisalle.edu.co 
para identificar puntos significativos de emisión y establecer estrategias para su reducción en pro de la mejora de la calidad del aire de los ciudadanos. Su desarrollo puede ser replicado en la generación de protocolos para la estimación de emisiones de fuentes móviles, considerando las ventajas que conIleva cuando existen limitaciones de información. El método propuesto aún cuenta con incertidumbres que no es posible determinar dada la calidad de las fuentes de información.

Palabras clave: contaminación atmosférica, desagregación espacio-temporal, modelación ambiental, modelación de calidad del aire.

\section{ABSTRACT}

Aim: This study presents the reconciliation of inventories of mobile emission in Bogota in 2013, applying top-down and bottom-up methodologies.

Method: A comparison of emissions from different vehicle categories was conducted, as well as the geographical and temporal distribution of them, by creating a processing code in JAVA. The process employed the largest amount of information available from the authorities of the Capital District, and previous studies. The innovation of this research is the estimation of the traffic flows in secondary roads, based on an algorithm that considers road geometry, interaction between arterial and secondary roads, and legal restrictions on traffic.

Results: The results show the estimated emissions for pollutants $\mathrm{CO}, \mathrm{CO}_{2}, \mathrm{VOCs}, \mathrm{NO}_{\mathrm{x}^{\prime}} \mathrm{SO}_{2}$ and $\mathrm{PM}$, and its comparison with other studies for the city. The values obtained can be classified as minimum and maximum references of the emissions generated in 2013, due to data uncertainty.

Conclusions: The project provides an upmost useful tool as input for the authorities in order to identify areas with significant emissions and to develop strategies for emissions reduction, towards improving the air quality of citizens. This method can be replicated in the generation of protocols for estimating emissions from mobile sources, but results need to be carefully reviewed given the quality of information sources.

Keywords: air pollution, air quality modeling, environmental modeling, spatio-temporal disaggregation.

\section{INTRODUCCIÓN}

Bogotá (Colombia) es una de las ciudades con mayor contaminación del aire en Suramérica, siendo el material particulado la causa de más preocupación (Cárdenas-Franco, 2012; OMS, 2014). Mientras que el problema ha mejorado en los últimos años $\left(\mathrm{PM}_{10}\right.$ promedio anual se redujo de $74 \mathrm{ug} / \mathrm{m}^{3}$ a $48 \mathrm{ug} / \mathrm{m}^{3}$ entre 2005 y 2013), aún ocurren concentraciones por encima de los estándares considerados seguros (la OMS establece un promedio anual de $40 \mathrm{ug} / \mathrm{m}^{3}$ para $\left.\mathrm{PM}_{10} ; \mathrm{OMS}, 2006\right)$, en especial al suroccidente de la ciudad (SDA, 2011; SDA, 2015a). Varios investigadores han señalado la afectación a la salud pública por la contaminación del aire bogotano, en especial la población más vulnerable: niños y tercera edad (Hernández et al., 2009; Arciniegas et al., 2006a, 2006b; Franco et al., 2009).
Estudios recientes apuntan hacia los vehículos como la principal fuente directa e indirecta de emisión de contaminantes al aire en Bogotá (Ingeniería DICTUC, 2006; Rodríguez y Behrentz, 2009; SDA, 2011; Rojas y Peñaloza, 2010; Beltrán, Belalcázar y Rojas, 2012; Pachón, 2013). Las emisiones de estas fuentes están asociadas a la calidad del combustible, patrones de conducción, condiciones geográficas, estado y mantenimiento de los vehículos y de las vías, velocidades promedio de circulación, entre otros. Los vehículos también influencian la generación de material particulado resuspendido en la atmósfera.

En el caso de la estimación de inventarios de emisiones para fuentes móviles, se aplican tradicionalmente metodologías top-down y bottom-up (Lents et al., 2011). Rojas y Peñaloza (2010) realizaron un inventario desagregado para la ciudad, 
mientras que otros trabajos previos de inventarios de emisiones no contemplaron su desagregación espacio-temporal, ni una conciliación entre metodologías top-down y bottom-up, lo que resulta en una herramienta de comparación y ajuste que permite tener un inventario de emisiones con una menor incertidumbre (Ossés de Eicker, Zah, Triviño y Hurni, 2008; Saide, Zah, Osses y Ossés de Eicker, 2009; Tuia et al., 2007) aunque es sabido que la aplicación de las dos metodologías, incluso con la misma información de entrada, puede llevar a diferentes resultados de emisiones (Wang et al., 2009).

\section{METODOLOGÍA}

\section{Inventario de emisiones top-down}

El enfoque top-down se refiere tradicionalmente a la construcción del inventario partiendo de valores de emisiones del dominio completo o de baja resolución (por ejemplo, a nivel país). La estimación de emisiones de fuentes móviles por contaminante y categoría vehicular se realiza empleando la ecuación (1) (US EPA, 1997).

$$
E_{i}=\sum_{j=0}^{n} \mathrm{FE}_{i j} * \mathrm{FA}_{j} * \mathrm{~N}_{j}
$$

Donde, $E_{\mathrm{i}}$ (g/año): emisiones totales del contaminante $i\left(\mathrm{PM}, \mathrm{CO}, \mathrm{CO}_{2}, \mathrm{NOx}, \mathrm{SOx}, \mathrm{VOCs}\right)$.

$\mathrm{FE}_{\mathrm{ij}}(\mathrm{g} / \mathrm{km}$-veh): factor de emisión del contaminante $i$ para la categoría vehicular $j$.

$\mathrm{FA}_{\mathrm{j}}(\mathrm{km} / \mathrm{año})$ : factor de actividad para la categoría vehicular $j$.

$\mathrm{N}_{\mathrm{j}}$ (veh): número de vehículos para la categoría vehicular $j$.

En este proyecto, se usaron los factores de emisión y los factores de actividad para 38 categorías vehiculares, recopilados de diferentes estudios (CAF, 2007; Rodríguez y Behrentz, 2009; Hernández-González y Jiménez, 2011; Rojas y Peñaloza, 2010; SDA, 2011; SDA y UNAL, 2013). De las categorías vehiculares, 32 coinciden con las empleadas en el Plan Decenal de Descontaminación del Aire de Bogotá (PDDAB) (SDA, 2011), dos más incluyen subdivisiones para la categoría de motos de cuatro tiempos considerando su cilindraje, y cuatro corresponden a buses articulados y biarticulados con tecnología de emisión de gases Euro IV y V. Se seleccionaron factores de emisión reportados con incertidumbres cuantitativas para propagarlas en la emisión final. Las incertidumbres para los factores de emisión no disponibles en la literatura, fueron estimadas por ponderación lineal de aquellas reportadas por Rojas y Peñaloza (2010).

Para la determinación del número de vehículos de cada categoría circulando en la ciudad, se utilizó la base de datos del Registro Distrital Automotor (RDA), proporcionada por la Secretaria Distrital de Movilidad (SDM), con corte a 31 de diciembre de 2013. Los datos se encontraban clasificados por año, modelo, cilindraje, tipo de servicio y combustible. Transmilenio S.A. (TM) proporcionó el número de buses vinculados al Bus Rapid Transit (BRT) y el Sistema Integrado de Transporte Público (SITP) a la misma fecha de corte, detallando año, modelo y clase de vehículo. Para las motos, la información fue obtenida de los resultados del Contrato Interadministrativo 013 de 2012, suscrito entre la Secretaría Distrital de Ambiente (SDA) y la Universidad Nacional (UN). Los factores de emisión y los factores de actividad utilizados se encuentran en el anexo 1.

\section{Inventario de emisiones bottom-up}

En la metodología bottom-up, el inventario se realiza tradicionalmente desde datos locales con mayor nivel de detalle o alta resolución, como flujos o volúmenes vehiculares, tipo y longitud de vías o perfiles horarios de tráfico. Las emisiones por contaminante y categoría vehicular se estiman aplicando la ecuación (2), que corresponde a la transformación de la ecuación (1) en función de las variables mencionadas y conservando los mismos factores de emisión en este caso.

$$
E_{i}=\sum_{j=0}^{n} \mathrm{FE}_{i j} * \mathrm{~F}_{j k} * \mathrm{~L}_{k}
$$


Donde, $\mathrm{E}_{\mathrm{i}}(\mathrm{g} / \mathrm{h})$ : emisiones totales del contaminante $i$ (PM, CO, $\mathrm{CO}_{2}, \mathrm{NOx}, \mathrm{SOx}$, VOCs).

$\mathrm{FE}_{\mathrm{ij}}(\mathrm{g} / \mathrm{km}-\mathrm{veh})$ : factor de emisión del contaminante $i$ para la categoría vehicular $j$.

$F_{j k}(v e h / h)$ : flujo vehicular para la categoría vehicular $j$ en la categoría de malla vial $k$.

$\mathrm{L}_{\mathrm{k}}(\mathrm{km})$ : longitud de la vía para la categoría de malla vial $k$.

En este proyecto las emisiones se desagregaron a un nivel de detalle por hora y en una resolución espacial de $1 \times 1 \mathrm{~km}^{2}$, en un dominio con una malla de $55 \times 55$ celdas. La superposición entre el dominio de modelación (cuadrícula) y la malla vial genera la subdivisión de las vías en segmentos viales, a los cuales es posible asignarles atributos como: categoría de vía (arterial, intermedia, local, residencia, rural), flujo vehicular (medido en el punto de aforo y extrapolado a la malla vial haciendo uso de los polígonos de Thiessen), y uso de suelo. Al finalizar, la emisión de cada celda corresponde a la sumatoria de los aportes de cada segmento vial incluidos en dicha celda.
La figura 1 muestra las actividades desarrolladas para la estimación de emisiones mediante la aproximación bottom-up. Con el fin de extraer la información de conteos vehiculares y poder distribuir las emisiones en el espacio y tiempo, se desarrolló un código de cálculo basado en matrices en JAVA, el cual almacena los datos de entrada, vincula sus atributos, asigna valores de restricción y realiza las operaciones de multiplicación y adición iterativamente.

\section{Malla vial}

La malla vial de Bogotá está conformada por el conjunto de vías arteriales, intermedias, locales y rurales (tabla 1). A esta red también se integra la infraestructura de vías de Transmilenio (sistema de transporte masivo de la ciudad de tipología BRT).

El cálculo efectivo de la longitud de los segmentos viales para cada tipo de vía se realizó usando ArcGIS $®$ 10.2. La información geográfica de la red de la malla vial correspondió a la versión 12.13 de diciembre de 2013, del Mapa de Referencia para

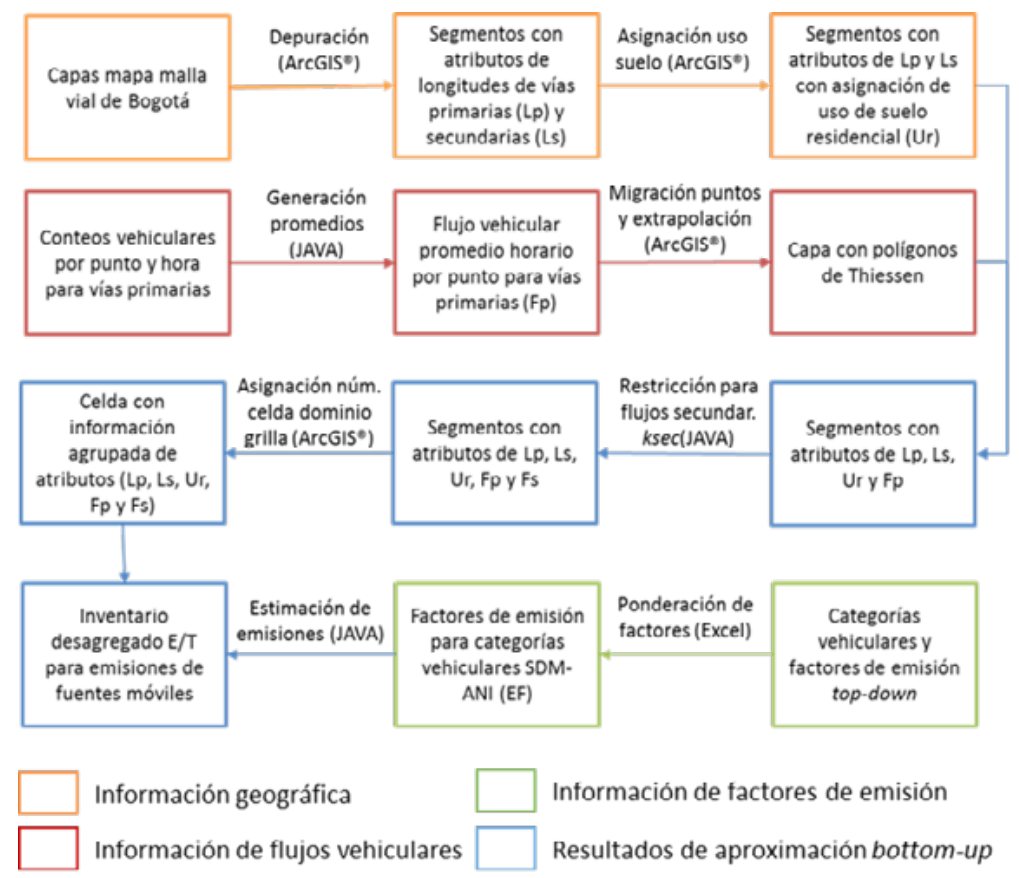

Figura 1. Flujograma para desagregación espacio temporal de emisiones por fuentes móviles

Fuente: elaboración propia. 
Tabla 1. Clasificación de la malla vial de la ciudad

\begin{tabular}{lcc}
\hline \multicolumn{1}{c}{ Tipo } & Tipo de clasificación & Tipologías especiales de vías según sección \\
\hline Primaria & Malla vial arterial & Vías de $100,60,40,30,28,25$ y 22 (en zona rural) metros de ancho \\
\hline Secundaria & Malla vial intermedia & Comprende vías de 22,18 y 16 metros \\
\hline Secundaria & Malla vial local & Comprende vías de 13,10 y 8 metros \\
\hline Secundaria & Malla vial rural & Vías que conectan o atraviesan el uso de suelo rural \\
\hline Transmilenio & Troncales Transmilenio & Carriles de uso exclusivos del sistema \\
\hline
\end{tabular}

Fuente: elaboración propia.

el Distrito Capital y del Catálogo de Datos Geográficos (UAECD, 2014).

\section{Flujo vehicular}

Para analizar el volumen vehicular de la ciudad en cuanto a horario, para diferentes tipos de vías y por categoría vehicular, se utilizó la información proporcionada para 40 intersecciones de la SDM (2014) para el año 2013, y de seis peajes de la Agencia Nacional de Infraestructura (ANI, 2013) para el 2012. Se cuantificó la demanda del tránsito vehicular, es decir, frecuencia y cantidad de vehículos, para los 46 puntos de monitoreo viales identificados en la figura 2. La metodología para el aforo vehicular de las intersecciones se encuentra definida por la SDM y C\&M (2005). Para el caso de los peajes, corresponde al tráfico promedio diario durante un mes de los proyectos de concesión carretero a cargo de la ANI.

Dado que la información de volúmenes vehiculares se encontraba sin procesar, fue necesario desarrollar un código en JAVA, que permitiera extraer la información de flujo de las bases de datos de la SDM y obtuviera el valor promedio por vía para un punto de monitoreo específico. La información se encontraba originalmente organizada por periodos de cada 15 minutos y por cada acceso de la vía, para posteriormente al procesamiento quedar consolidada en valores horarios y por el total de la vía.

Toda vez que los aforos se realizan únicamente sobre 46 puntos en vías arteriales de la ciudad, fue necesario extrapolar la información de tráfico al resto de vías haciendo uso del método de polígonos de Thiessen en $\operatorname{ArcGIS}^{\circledR}$. Esta técnica de interpolación es ampliamente utilizada para la desagregación de inventarios dada su simplicidad, efectividad y aplicabilidad para el área objeto de estudio (Denby et al., 2005; Horalek et al., 2005). Se utilizó este resultado para asignar el flujo sobre las demás vías arteriales en donde no se realizaron mediciones. El método define polígonos

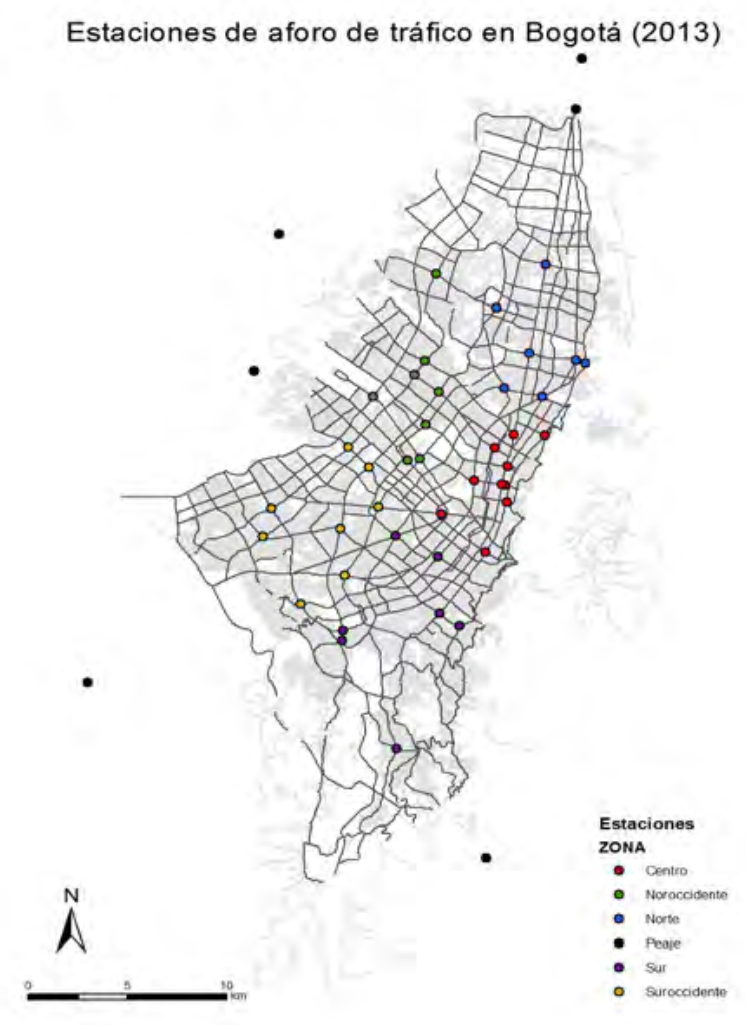

Figura 2. Localización puntos de monitoreo de tráfico de la SDM y peajes de la ANI

Fuente: elaboración propia con información de SDM. 
determinados por las mediatrices a los segmentos de unión de los puntos de aforo, de forma que su perímetro es equidistante a los puntos vecinos (US EPA, 2004). Esta metodología complementa los avances realizados por Rojas y Peñaloza (2010), al incluir un mayor número de puntos de aforo y una asignación de valores de flujo vehicular específicos de acuerdo con la categoría de las vías.

Debido a la carencia de información de flujo vehicular en vías secundarias de la ciudad, se tuvieron en cuenta las siguientes consideraciones con el propósito de estimar su magnitud:

i. La actividad vehicular de las vías secundarias no podrá ser igual o mayor a la de las vías principales, considerando que estas últimas son alimentadas por las secundarias. Para lo cual se definió un factor $\left(\mathrm{k}_{\mathrm{sec}}\right)$ con valores entre cero y uno, de acuerdo con la ecuación (3).

$$
k_{\text {sec }}=\frac{F_{\text {sec }} \cdot L_{\text {sec }}}{F_{\text {art }} \cdot L_{\text {art }}}
$$

Donde, ksec: factor que limita la actividad vehicular de las vías secundarias por debajo de la actividad vehicular de las vías arteriales de una celda $(0>$ ksec $\leq 1)$.

Fsec: flujo vehicular de las vías secundarias que pasa por la celda (veh/h).

Lsec: longitud de las vías secundarias contenidas la celda $(\mathrm{km})$.

Fart: flujo vehicular de las vías principales (arteriales) que pasa por la celda (veh/h).

Lart: longitud de las vías arteriales contenidas la celda $(\mathrm{km})$.

ii. El flujo de las vías secundarias se ve directamente afectado por la capacidad de las vías. Por configuración geométrica una vía secundaria comúnmente es más angosta que una vía arterial (tabla 1), y por tanto el volumen de vehículos se verá reducido en la misma proporción. De aquí se obtiene que para una vía intermedia el valor es de 0,37 , obtenido como la relación entre el ancho promedio de una vía intermedia (22 m) respecto al ancho promedio de una vía arterial (60 m). Para las vías locales (ancho promedio de $13 \mathrm{~m}$ ) el valor será de 0,22.

iii. Para la malla vial local con uso residencial se establece un valor de cero (0) para las categorías de camiones grandes y transporte público colectivo bus por restricción legal.

iv. Para la malla vial Transmilenio se mantiene el valor de flujo de buses articulados obtenido mediante la distribución por polígonos de Thiessen.

\section{Factores de emisión}

En razón a que la SDM tiene definidas 15 categorías vehiculares para realizar los aforos vehiculares en la ciudad y la ANI cuenta con siete, lo que difiere de las 38 establecidas en la metodología topdown según la información del PDDAB, mediante la ecuación (4) fue necesario obtener un factor de emisión ponderado para cada categoría SDM, en función del número de vehículos registrados del parque automotor.

$$
\begin{gathered}
F E_{\text {PONDERADO-CAT SDM-ANIj }}= \\
\frac{\sum_{a=1}^{n}\left(\mathrm{FE}_{\mathrm{CAT}-\mathrm{a} \text { PDDAB }} * \mathrm{~N}_{\mathrm{CAT}-\mathrm{a} \text { PDDAB }}\right)}{\sum_{a=1}^{n}\left(\mathrm{~N}_{\mathrm{CAT}-\mathrm{a} \text { PDDAB }}\right)}
\end{gathered}
$$

Donde, $\mathrm{FE}_{\text {PONDERADO-CAT SDM-ANIj }}(\mathrm{g} / \mathrm{km}$-veh): factor de emisión ponderado del contaminante $i$ para la categoría vehicular agrupada $j$ de la SDM.

$\mathrm{FE}_{\text {CAT-a PDDAB }}(\mathrm{g} / \mathrm{km}$-veh): factor de emisión del contaminante $i$ para una categoría vehicular individual a del PDDAB.

$\mathrm{N}_{\text {CAT-a PDDAB }}$ (veh): número de vehicular de la categoría a del PDDAB.

Los factores de emisión y sus incertidumbres fueron igualmente recopilados de diferentes referencias bibliográficas (CAF, 2007; Rodríguez y Behrentz, 2009; Rojas y Peñaloza, 2010; SDA, 2011; Hernández-González y Jiménez, 2011; SDA y UNAL, 2013). 


\section{RESULTADOS}

\section{Inventario general de emisiones top-down}

La estimación top-down es calculada para la emisión anual de los diferentes contaminantes para cada categoría vehicular definida. El número de vehículos, resultado de la consolidación de las diferentes bases de datos (SDM, TM, SDA), se incluye en el anexo 1 (disponible en: https://drive.google.com/ open?id=0B3lzbQba8UcELTVwc0tMYjhqR1U). La tabla 2 presenta los estimativos del inventario de emisiones de fuentes móviles para el año 2013, distribuidos por categorías vehicular.

Las categorías de mayor aporte al inventario de PM son los camiones (C-36,4\%), buses de transporte público (B-29,1\%), y con similar participación las motos (M-7,4\%) y microbuses (MB-7,1 $\%)$. Por su parte, las categorías de mayor aporte de $\mathrm{NO}_{x}$ son los vehículos particulares (VP-23,4\%) seguido por camperos/camionetas (CC-18,9\%), taxis (T-18,3\%) y camiones (C-12,4\%). En cuanto a las emisiones de los restantes contaminantes VOC, $\mathrm{CO}, \mathrm{SO}_{2}$ y $\mathrm{CO}_{2}$ los vehículos particulares presentan el mayor porcentaje de aporte con niveles del 38,5\%, $34 \%, 64 \%$, y 38,2 \%, respectivamente. Las motocicletas $(\mathrm{M})$ generan su mayor aporte a las emisiones de CO y VOC. Las categorías Transmilenio (TM) y Transporte Especial y de Turismo (ET) se caracterizan por generar bajos niveles de aporte de emisión para los contaminantes estudiados.

\section{Inventario de emisiones bottom-up: desagregación espacial y temporal}

\section{Malla vial}

De acuerdo con el análisis de la malla vial de Bo-

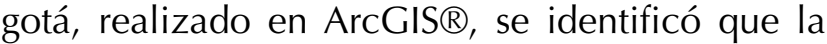
ciudad cuenta con $8276 \mathrm{~km}$-carril para el tipo de vías y la grilla evaluada, de los cuales, el $11 \%$ corresponden a malla vial arterial, $18 \%$ a intermedia, $63 \%$ local, $7 \%$ rural y $1 \%$ a las troncales de Transmilenio. Al finalizar el procesamiento de la información cartográfica, se obtuvo la información de todos los tramos (aprox. 131.071 segmentos en la malla vial de Bogotá), cada uno de los cuales fue asociado a una celda del dominio.

Tabla 2. Total inventario general de emisiones por contaminante e incertidumbres (ton/año) por categoría vehicular

\begin{tabular}{ccccccccccccc}
\hline \multirow{2}{*}{$\begin{array}{c}\text { Categoría } \\
\text { vehicular }\end{array}$} & \multicolumn{2}{c}{$\mathbf{C O}_{\mathbf{2}}$} & \multicolumn{2}{c}{$\mathbf{C O}$} & \multicolumn{2}{c}{$\mathbf{N O}_{\mathbf{x}}$} & \multicolumn{2}{c}{ VOC } & \multicolumn{2}{c}{$\mathbf{P M}$} & \multicolumn{2}{c}{$\mathbf{S O}_{2}$} \\
\hline & Valor & $\mathbf{I}$ & Valor & $\mathbf{I}$ & Valor & $\mathbf{I}$ & Valor & $\mathbf{I}$ & Valor & $\mathbf{I}$ & Valor & $\mathbf{I}$ \\
\hline $\mathrm{VP}$ & 3.998 .558 & 401.129 & 294.970 & 119.904 & 15.550 & 7.764 & 35.418 & 15.657 & 45 & 31 & 9.025 & 3.131 \\
\hline $\mathrm{CC}$ & 2.880 .139 & 642.771 & 196.222 & 91.727 & 12.567 & 6.348 & 19.806 & 6.377 & 118 & 81 & 1.875 & 670 \\
\hline $\mathrm{T}$ & 966.706 & 137.013 & 41.865 & 13.701 & 12.179 & 4.948 & 12.940 & 4.187 & 3 & 2 & 1.324 & 457 \\
\hline $\mathrm{M}$ & 1.051 .331 & 205.325 & 258.587 & 150.040 & 5.417 & 3.262 & 17.814 & 5.454 & 86 & 46 & 692 & 244 \\
\hline $\mathrm{B}$ & 501.859 & 90.335 & 27.061 & 10.277 & 6.868 & 3.235 & 3.528 & 1.244 & 338 & 255 & 442 & 151 \\
\hline $\mathrm{TM}$ & 221.189 & 39.814 & 1.116 & 424 & 2.676 & 1.259 & 103 & 36 & 45 & 31 & 216 & 74 \\
\hline MB & 239.835 & 43.170 & 20.311 & 7.717 & 2.755 & 1.295 & 901 & 317 & 83 & 53 & 93 & 33 \\
\hline $\mathrm{ET}$ & 26.319 & 4.737 & 421 & 160 & 301 & 142 & 97 & 34 & 23 & 15 & 21 & 7 \\
\hline $\mathrm{C}$ & 572.286 & 103.012 & 25.891 & 9.839 & 8.227 & 3.872 & 1.279 & 452 & 423 & 251 & 420 & 145 \\
\hline Total & 10.458 .221 & 1.667 .306 & 866.445 & 403.789 & 66.540 & 32.124 & 91.885 & 33.757 & 1.163 & 803 & 14.109 & 4.911 \\
\hline
\end{tabular}

Nota: I - incertidumbre, C-camiones, ET-transporte especial y de turismo, TM-Transmilenio, B-buses, MB-microbuses, M-motos, T-taxis, CC-camperos y camionetas, VP-vehículos particulares.

Fuente: elaboración propia. 


\section{Flujo vehicular}

Las figuras 3 y 4 presentan el comportamiento del flujo vehicular diario con relación a los 40 puntos monitoreados por la SDM. Los flujos horarios por vía se distribuyen en $6,4 \%$ para volúmenes vehiculares menores a $800 \mathrm{veh} / \mathrm{hora} ; 56,1 \%$ para flujos entre 800 y 6000 veh/hora, $23,3 \%$ para flujos entre 6000 y $11.000 \mathrm{veh} / \mathrm{hora}$ y $14,2 \%$ para flujos mayores a $11.000 \mathrm{veh} / \mathrm{hora}$. A partir de las 6:00 hasta las 19:00 horas se presenta el mayor tráfico vehicular. Se evidencia la mayor preponderancia que tienen los vehículos ligeros (automóviles particulares, camionetas, camperos y taxis) en la circulación urbana, con una proporción de 65,7\% para día hábil y 74 \% para día festivo; seguido de

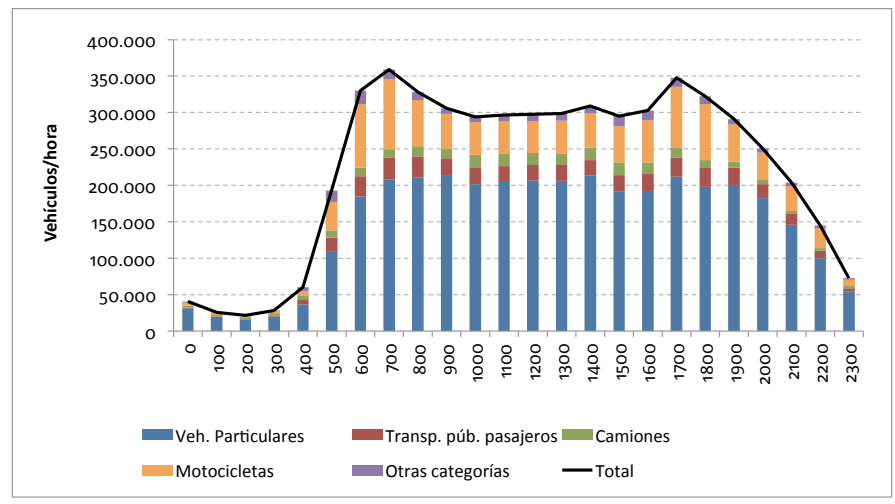

las motocicletas con proporciones de 18,5\% (hábil) y $13,3 \%$ (sábado).

\section{Factores de emisión}

En el anexo 2 se presentan los factores de emisión ponderados para las 15 categorías vehiculares que fueron cuantificadas mediantelos aforos vehiculares (disponible en: https://drive.google.com/open?id=0B3lzbQba8UcEU1JacDhZVkxQdzQ). El cálculo tambiénfueaplicadoa losfactores de incertidumbre.

\section{Inventario desagregado espacial y temporalmente}

La tabla 3 presenta el resultado de la ejecución del código JAVA para la obtención de las emisiones de

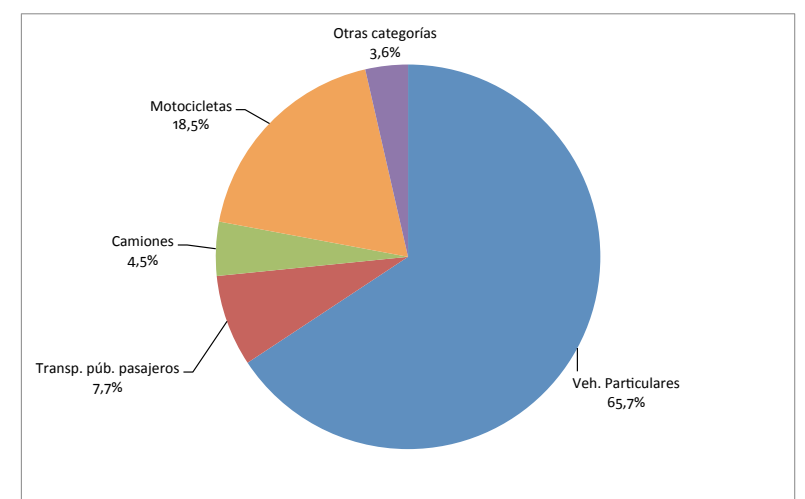

Figura 3. Perfil flujo horario y distribución categorías vehiculares por categoría para las 40 estaciones en día hábil Fuente: elaboración propia.
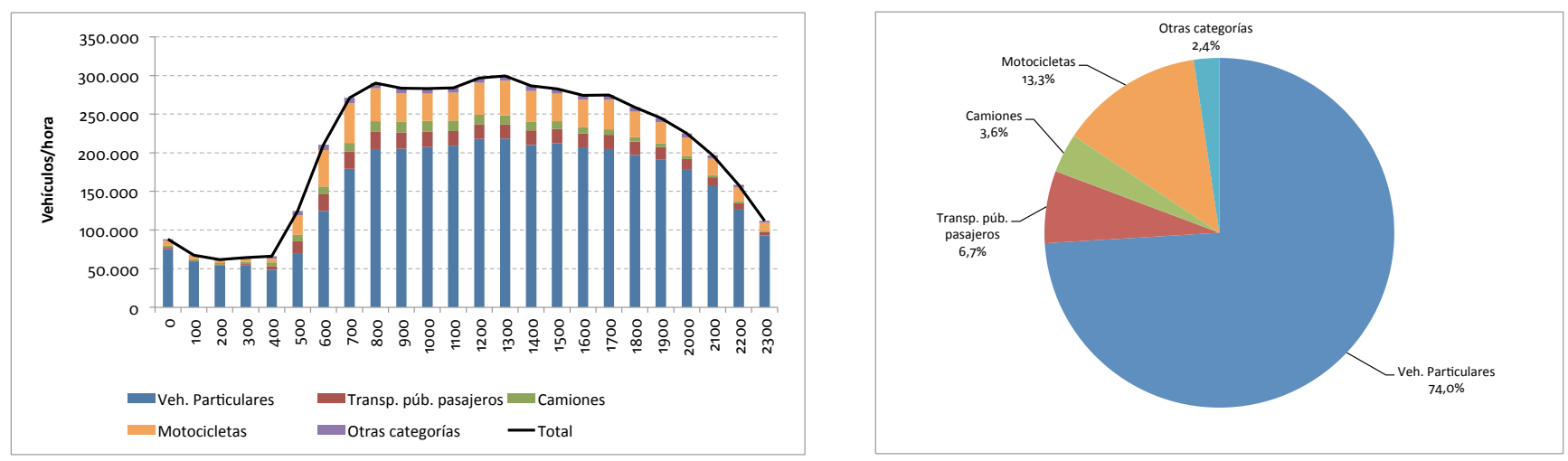

Figura 4. Perfil flujo horario y distribución categorías vehiculares por categoría para las 40 estaciones en día sábado Fuente: elaboración propia. 
fuentes móviles consolidando el resultado para los dos tipos de días típicos.

Tabla 3. Emisiones de fuentes móviles para la metodología bottom-up (ton/día).

\begin{tabular}{ccccc}
\hline \multirow{2}{*}{ Contaminante } & \multicolumn{2}{c}{ Hábil } & \multicolumn{2}{c}{ Sábado } \\
\cline { 2 - 5 } & Valor & Incert. & Valor & Incert. \\
\hline $\mathrm{CO}$ & 1.945 & 887 & 1.966 & 870 \\
\hline $\mathrm{CO}$ & 19.656 & 3.500 & 19.850 & 3.448 \\
\hline $\mathrm{COV}$ & 200 & 77 & 209 & 81 \\
\hline $\mathrm{MP}$ & 3,9 & 2,8 & 2,8 & 2,1 \\
\hline $\mathrm{NO}_{x}$ & 136 & 72 & 126 & 67 \\
\hline $\mathrm{SO}_{2}$ & 32 & 11 & 35 & 12 \\
\hline
\end{tabular}

Fuente: elaboración propia.
Se observa que no hay mayor diferencia entre los resultados obtenidos para el día hábil respecto al sábado. Si bien, en la tabla 3 se muestra el consolidado de las 24 horas, las figuras 3 y 4 permiten identificar que el patrón de flujos de tráfico varía, es decir, mientras que para día hábil el comportamiento es casi constante a partir del inicio de la hora pico a las 06:00 hasta las 08:59 (tipo meseta), para el día sábado la tendencia se concentra más sobre las horas del mediodía (tipo montaña). Adicionalmente, para los días hábiles se presenta restricción vehicular por el "pico y placa", lo que reduce el volumen de vehículos particulares en operación; mientras que para el día sábado esta medida no existe. Por su parte, la presencia de

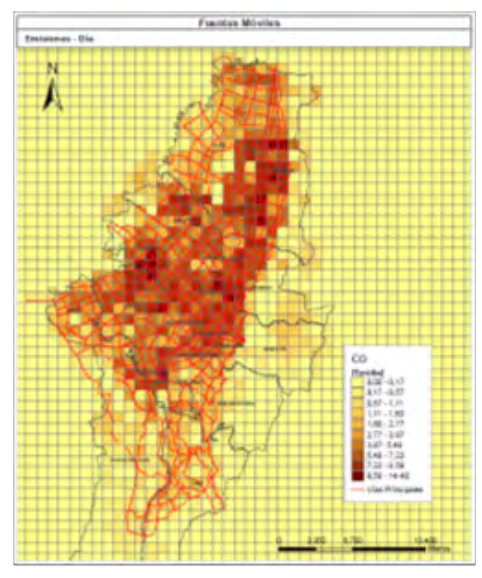

a) $\mathrm{CO}$

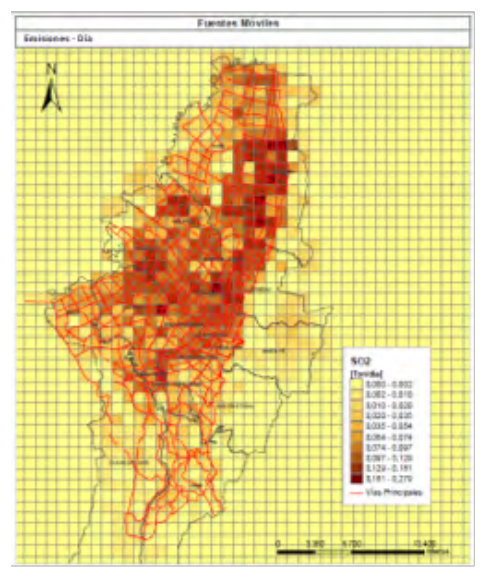

d) $\mathrm{SO}_{2}$

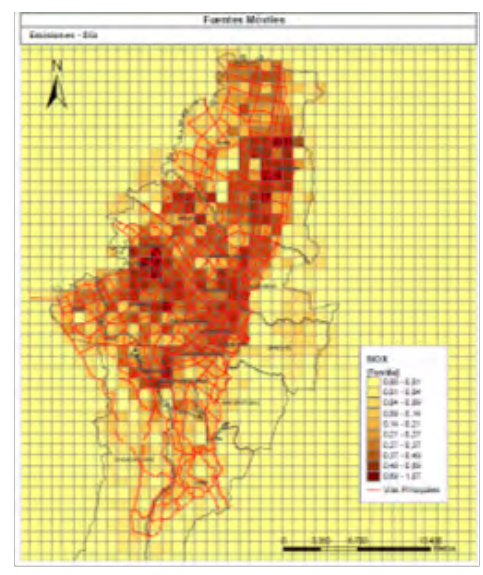

b) $N O x$

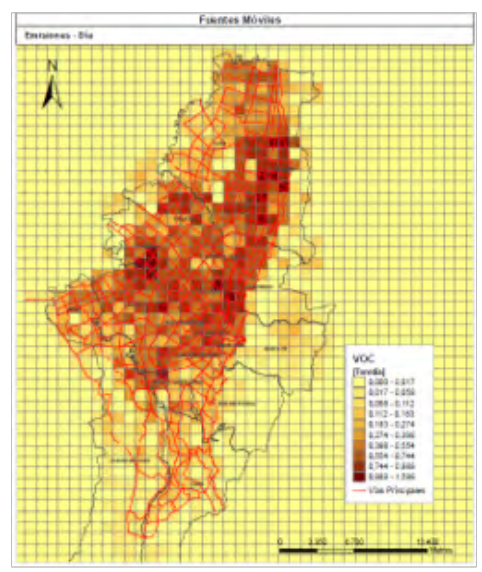

e) $\mathrm{CO}$

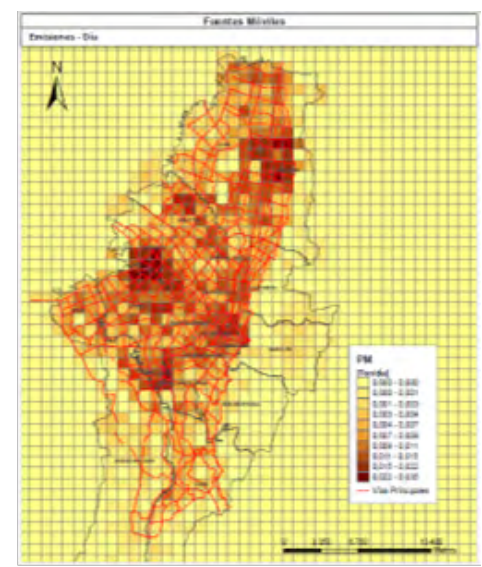

c) $P M$

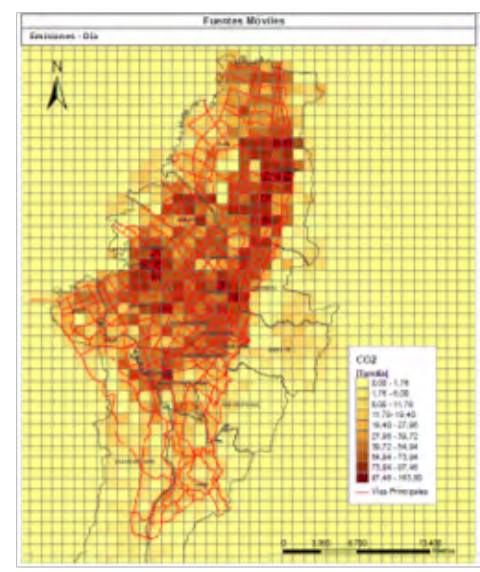

f) $\mathrm{CO}_{2}$

Figura 5. Desagregación espacio temporal para fuentes móviles para 24 horas en día hábil

Fuente: elaboración propia. 
buses, busetas y camiones en fines de semana se reduce, lo que genera el efecto de disminución en el material particulado (tabla 3).

La figura 5 presenta la desagregación espacial del inventario de emisiones bottom-up para los contaminantes $\mathrm{CO}, \mathrm{NO}_{x^{\prime}}, \mathrm{PM}, \mathrm{SO}_{2}, \mathrm{VOC}, \mathrm{CO}_{2}$ evaluados para el día hábil y una hora específica del día. Para todos los compuestos analizados, los mayores puntos de generación de emisión se concentran en ocho zonas donde se contabilizó un mayor volumen vehicular. El aumento en el número de puntos de conteo vehicular, pasando de 26 en 2008 a 40 en 2013, permitió refinar la estimación de los inventarios de fuentes móviles a nivel espacial. De esta forma se evidenció la presencia de nuevas áreas significativas de emisiones de contaminantes especialmente en el sur y norte de la ciudad. Lo anterior permite la adopción de medidas con un carácter preventivo y enfocadas a la reducción en la fuente, así como establecer estrategias de seguimiento y control más efectivas (García, García y Vaca, 2012). Así mismo, al comparar los mapas en los que las emisiones por vehículos particulares, camperos y camionetas son mayores $\left(\mathrm{CO}\right.$ y $\left.\mathrm{SO}_{2}\right)$, respecto a mapas en los que otras categorías vehiculares presentan una mayor contribución a diferentes contaminantes (por ejemplo, buses, busetas y camiones para PM), se observa como algunas zonas son más representativas en función del flujo vehicular específico al que se le deba atribuir la mayor emisión.

\section{Conciliación de inventarios de emisiones atmosféricas por fuentes móviles}

En la tabla 4 se presenta la comparación entre el inventario de emisiones atmosféricas por fuentes móviles top-down respecto a la estimación de emisiones anuales bottom-up. La conciliación se realiza en términos de emisiones totales al año, pues a su vez esta permite la comparación con estudios previos. Por su parte, la desagregación espacial y temporal del inventario top-down requiere de un criterio de distribución de información (surrogates), situación que no fue aplicada en el presente proyecto. El resultado de la aproximación bottom-up muestra un valor inferior en todos los contaminantes, con excepción del material particulado. Esta tasa menor de emisión es resultado de la menor actividad vehicular horaria de diversas categorías vehiculares calculada en el método bottom-up, especialmente en motocicletas (tabla 5). Sin embargo, para material particulado existe un aumento en el volumen vehicular en las categorías que generan un mayor aporte de este contaminante (buses y camiones). En todo caso, y con excepción del $\mathrm{CO}_{2}$, los valores totales anuales de las dos metodologías se consideran cercanos teniendo en cuenta que se traslapan con los rangos de incertidumbres de las diferentes metodologías. Por ejemplo, la incertidumbre de $\mathrm{CO}$ es alrededor del 46 $\%$, mientras que la diferencia obtenida entre las dos metodologías es de $17 \%$.

Tabla 4. Comparación de resultados bottom-up vs. top-down

\begin{tabular}{cccccc}
\hline \multirow{2}{*}{ Contaminante } & \multicolumn{2}{c}{$\begin{array}{c}\text { Desagregación (botton-up) } \\
\text { ton/año }\end{array}$} & \multicolumn{2}{c}{$\begin{array}{c}\text { Inv. general (top-down) } \\
\text { ton/año }\end{array}$} & \multirow{2}{*}{ Diferencia } \\
\cline { 2 - 5 } & Valor & Incert. & Valor & Incert. & \\
\hline $\mathrm{CO}$ & 717.945 & 321.110 & 866.445 & 403.789 & $-17 \%$ \\
\hline $\mathrm{CO}$ & 7.254 .350 & 1.268 .548 & 10.458 .221 & 1.667 .306 & $-31 \%$ \\
\hline $\mathrm{COV}$ & 74.579 & 28.450 & 91.885 & 33.757 & $-19 \%$ \\
\hline $\mathrm{MP}$ & 1.327 & 956 & 1.163 & 763 & $14 \%$ \\
\hline $\mathrm{NO}_{x}$ & 48.927 & 25.770 & 66.540 & 32.124 & $-26 \%$ \\
\hline $\mathrm{SO}_{2}$ & 12.085 & 4.258 & 14.109 & 4.911 & $-14 \%$ \\
\hline
\end{tabular}

Fuente: elaboración propia. 
La tabla 5 muestra un comparativo entre la cantidad de vehículos registrados en el distrito de acuerdo con el RDA, el factor de actividad por cada categoría vehicular (tanto top-down como bottom-up) y la representatividad de cada categoría con relación a los flujos aforados en las estaciones de monitoreo.

Se observa un incremento significativo en el flujo de buses y camiones durante los conteos vehiculares (bottom-up) respecto a la flota registrada oficialmente y frente a su correspondiente factor de actividad manejado en la metodología top-down. Esta se identifica como la principal causa del incremento del material particulado. Además, se aprecia una reducción significativa en la operación de las motos y de buses de Transmilenio desde la perspectiva bottom-up con relación a la aproximación top-down. Dado que los conteos vehiculares solo se realizan en vías principales, existe una incertidumbre aún no calculada para el flujo vehicular de vías secundarias que puede influir en el inventario de emisiones desde la metodología bottom-up.

Si bien era de esperarse una concordancia entre los valores de emisión estimados por las aproximaciones bottom-up y top-down; sin embargo, diversos factores incidieron en la diferencia entre estos dos resultados. La variación en los valores de estimación de emisiones en las dos metodologías se debió a diversas limitaciones que existen en las metodologías, que para el caso en particular se señalan en la tabla 6.

Tabla 5. Comparación de variables de movilidad para las dos metodologías evaluadas

\begin{tabular}{lccccc}
\hline \multicolumn{1}{c}{ Categoría vehicular } & $\begin{array}{c}\text { Vehículos } \\
\text { registrados al 2013 } \\
\text { (núm. vehículos) }\end{array}$ & $\mathbf{\%}$ & $\begin{array}{c}\text { Actividad vehicular } \\
\text { top-down } \\
\text { (veh-km/día) }\end{array}$ & \% & $\begin{array}{c}\text { Actividad vehicular } \\
\text { bottom-up } \\
\text { (veh-km/día) }\end{array}$ \\
\hline Automóviles, camperos y taxis & 1.446 .618 & $76,0 \%$ & 2.882 .974 & $66,3 \%$ & 2.907 .595 \\
\hline Microbús & 11.666 & $0,6 \%$ & 87.495 & $2,0 \%$ & 88.047 \\
\hline Bus, buseta e intermunicipales & 15.354 & $0,8 \%$ & 115.155 & $2,6 \%$ & 193.976 \\
\hline Buses articulados y biarticulados & 1.609 & $0,1 \%$ & 13.776 & $0,3 \%$ & 1.051 \\
\hline Buses alimentadores & 825 & $<0,1 \%$ & 6.531 & $0,2 \%$ & 9.798 \\
\hline Buses especiales & 608 & $<0,1 \%$ & 4.373 & $0,1 \%$ & 57.988 \\
\hline Camiones & 39.543 & $2,1 \%$ & 140.048 & $3,2 \%$ & 145.417 \\
\hline Motocicletas & 385.158 & $20,3 \%$ & 1.099 .195 & $25,3 \%$ & 493.571 \\
\hline Total & 1.901 .381 & $100 \%$ & 4.349 .546 & $100 \%$ & 3.897 .442 \\
\hline
\end{tabular}

Fuente: elaboración propia.

Tabla 6. Limitaciones en la estimación de emisiones

\begin{tabular}{|c|c|c|}
\hline Variable & Metodología & Limitaciones \\
\hline $\begin{array}{l}\text { Categoría } \\
\text { vehicular }\end{array}$ & $\begin{array}{l}\text { top-down } \\
\text { bottom-up }\end{array}$ & $\begin{array}{l}\text { Falta de homogenización de las clasificaciones vehiculares entre los distintos entes administrativos } \\
\text { que requieran información de flujos vehiculares (p. ej., para PDDAB son } 32 \text { mientras que para } \\
\text { SDM son 15). }\end{array}$ \\
\hline & & $\begin{array}{l}\text { Desactualización de factores de emisión propios para la ciudad debido a recientes cambios en la } \\
\text { calidad del combustible. }\end{array}$ \\
\hline $\begin{array}{l}\text { Número } \\
\text { de vehícu- } \\
\text { los }\end{array}$ & top-down & $\begin{array}{l}\text { Ausencia de un reporte obligatorio a las autoridades de los procesos de reconversión a gas natural, } \\
\text { híbrido o cualquier otra tecnología de combustible, desactualizando las bases de datos oficiales. } \\
\text { Omisión del número de vehículos no pertenecientes a la ciudad de Bogotá. }\end{array}$ \\
\hline & top-down & bre al estimar el factor de actividad de cada categoría vehicula \\
\hline $\begin{array}{l}\text { Flujo vehi- } \\
\text { cular }\end{array}$ & bottom-up & $\begin{array}{l}\text { Carencia de aforos vehiculares en otras tipologías de vía no arteriales, como intermedias, locales y } \\
\text { rurales, reduciendo la certeza en la estimación de la actividad vehicular de la malla secundaria y } \\
\text { sus respectivas subtipologías. } \\
\text { Falta de segregación de taxis por combustible al momento de ejecutar los aforos imposibilitando } \\
\text { la opción de evaluar su impacto de forma independiente. } \\
\text { Ausencia de un modelo de tráfico de la ciudad con el fin de tener una aproximación más detallada } \\
\text { del comportamiento y distribución de los vehículos a lo largo el día y los diferentes tipos de vías. }\end{array}$ \\
\hline Longitud & bottom-up & Restricciones en la información cargada a la geo-database, por ejemplo, ancho de la vía. \\
\hline
\end{tabular}

Fuente: elaboración propia. 


\section{Comparación del inventario general de emisiones con otros trabajos}

En la tabla 7 se muestran los resultados obtenidos respecto a otros estudios realizados para la ciudad. Se observa que los valores son cercanos a los obtenidos por la SDA (2014), los cuales se pueden considerar coherentes, debido a que la flota vehicular se ha incrementado un $69 \%$ desde 2008, especialmente en las categorías de automóviles y motocicletas. De hecho, entre 2002 y 2012 la flota de vehículos particulares, camperos y camionetas en la ciudad aumentó en un $200 \%$, teniendo en la actualidad cerca de 1.400 .000 unidades, mientras que los buses, camiones y taxis se han mantenido estables o incluso se han reducido. Por su parte, las motos también muestran incremento del $63 \%$ respecto al año 2008 (SDA 2015b), incidiendo directamente en las emisiones de CO y VOC. El gas natural vehicular ha venido reemplazando la gasolina, especialmente en autos particulares de gran cilindraje, camperos, camionetas y taxis (Minminas, 2014), lo que se refleja en mayores emisiones de NOx y VOC por parte de estas categorías vehiculares con relación al total del año 2008.

Al comparar las emisiones de PM del servicio público colectivo dentro del PDDAB para el 2008 con respecto a la actualización en 2013, se encuentra que las emisiones se han mantenido constantes, con una tendencia a la baja. Lo anterior se explica por la reducción del parque automotor de servicio público colectivo por la entrada del SITP, contando en el 2008 con un parque de 17.536 vehículos y en 2013 de 16.011, según el Observatorio Ambiental de Bogotá (SDA 2015c).

Frente a la validez de los resultados, este estudio se mantiene dentro del rango de máximos y mínimos obtenidos en otras publicaciones, y se podría establecer que las emisiones del año 2013 se encuentran dadas por el rango obtenido entre los valores bottom-up y top-down.

Se identifica que los resultados muestran diferencias significativas con los valores reportados por Rojas y Peñaloza (2010); sin embargo, fuera del año evaluado, las metodologías utilizadas difieren, puesto que en dicho estudio se realiza una combinación top-down y bottom-up, además de

Tabla 7. Comparación de inventarios de emisiones en Bogotá

\begin{tabular}{|c|c|c|c|c|c|c|c|}
\hline \multirow{2}{*}{ Estudio } & \multirow{2}{*}{$\begin{array}{c}\text { Año } \\
\text { evaluado }\end{array}$} & \multicolumn{6}{|c|}{ Emisión (Ton/Año) } \\
\hline & & $\mathrm{CO}_{2}$ & $\mathrm{CO}$ & NOx & VOC & PM & $\mathrm{SO}_{2}$ \\
\hline Este estudio, bottom-up & 2013 & $7,254,350$ & 717,945 & 48,927 & 74,579 & 1,327 & 12,085 \\
\hline Este estudio, top-down & 2013 & $10,458,221$ & 866,445 & 66,540 & 91,885 & 1,163 & 14,109 \\
\hline SDA (2014) & 2013 & $10,232,234$ & 741,560 & 62,918 & 65,247 & 836 & NA \\
\hline $\begin{array}{l}\text { Hernández-González } \\
\text { y Jiménez (2011) }\end{array}$ & 2008 & $3,160,896$ & NA & NA & NA & NA & NA \\
\hline Rojas y Peñaloza (2010) & 2008 & NA & 706,932 & 57,658 & 108,011 & 1,594 & 13,009 \\
\hline SDA y UDC (2011) & 2010 & NA & NA & NA & 48,350 & NA & NA \\
\hline SDA (2011) & 2008 & $6,000,000$ & 490,000 & 54,000 & 62,000 & 1,400 & $\mathrm{NA}$ \\
\hline $\begin{array}{l}\text { Rodríguez y Beherentz } \\
(2009)\end{array}$ & 2008 & $4,800,000$ & 450,000 & 30,000 & 60,000 & 1,100 & NA \\
\hline Promedio & - & $6,984,284$ & 662,147 & 53,340 & 72,868 & 1,237 & 13,068 \\
\hline Mínimo & - & $3,160,896$ & 450,000 & 30,000 & 48,350 & 836 & 12,085 \\
\hline Máximo & - & $10,458,221$ & 866,445 & 66,540 & 108,011 & 1,594 & 14,109 \\
\hline
\end{tabular}

Fuente: elaboración propia. 
hacer supuestos muy distintos a los de la presente estimación, especialmente para los flujos vehiculares en vías principales y secundarios. Por ejemplo, el volumen de las vías secundarias se obtuvo mediante consulta a personas del sector transporte, mientras que en el presente estudio se estableció un factor en función de la capacidad vial y al concepto de balance de masa. Por otra parte, en Rojas y Peñaloza (2010) los flujos tomados fueron totales del punto y no promedio de la vía.

\section{CONCLUSIONES}

Se realizó la conciliación de inventarios de emisiones de fuentes móviles bajo las aproximaciones top-down y bottom-up. Esto fue realizado mediante la comparación de los resultados totales de ambas metodologías y su respectivo análisis de las incertidumbres de las estimaciones. Se encontró una similitud en los valores de emisiones obtenidos, lo que a su vez se estableció como un rango en el inventario de emisiones para la ciudad. De acuerdo con los valores obtenidos, para el año 2013 las fuentes vehiculares en Bogotá emitieron aproximadamente: $7^{\prime} 000.000$ a $10^{\prime} 500.000$ ton $\mathrm{CO}_{2} ; 700.000$ a 870.000 ton $\mathrm{CO} ; 48.000$ a 67.000 ton de $\mathrm{NO}_{x} ; 74.000$ a 92.000 ton $\mathrm{VOC}, 1.100 \mathrm{a}$ 1.300 ton de PM y 12.000 a 14.000 ton $\mathrm{SO}_{2}$. El estudio fue comparado respecto a otras publicaciones de inventario de fuentes móviles para Bogotá, identificando la razonabilidad de los resultados del presente estudio.

Se establecieron metodologías para la estimación de flujos vehiculares en vías secundarias, procesamiento de la geo-database, desagregación espacio-temporal de las emisiones, entre otras, las cuales pueden ser usadas a futuro para actualizar el inventario de emisiones de forma periódica. Se generaron perfiles diarios de tráfico a partir de la información de conteos vehiculares que fueron usados en la desagregación temporal de las emisiones.
Se desarrolló e implementó una herramienta en código JAVA (disponible en: https://github.com/SDA-SALLE/excelParser), para la extracción y procesamiento de información de flujos vehiculares, así como su asociación a los respectivos factores de emisión. La herramienta también permite el análisis y ponderación de datos para cálculo de flujo vehicular en vías secundarias, las cuales no contaban con mediciones en estudios anteriores.

La distribución geográfica de las emisiones permitió distinguir nuevas zonas críticas en el norte y sur de la ciudad que no habían sido identificadas en estudios previos por la falta de información más detallada de aforos de tráfico, lo cual sirve de herramienta de planificación y seguimiento para las autoridades de la ciudad.

La estimación de emisiones posee una alta sensibilidad al método de tratamiento de los datos de conteo vehicular. De igual forma, limitaciones relacionadas con la homogenización de la información, desactualización de factores de emisión, ausencia de aforos vehiculares en vías secundarias, restricciones en la información de la geo-database, entre otras (tabla 6), incrementaron el nivel de incertidumbre de los resultados, por lo que se hace necesario contar con un modelo de tráfico en la ciudad que responda a estas inquietudes. Los hallazgos identificados con el estudio pueden ser empleados por las autoridades locales como instrumento para la planificación de la movilidad y el control de la contaminación del Distrito Capital, desafíos claros para mejorar la calidad de vida de los ciudadanos.

\section{FINANCIAMIENTO}

Este proyecto fue financiado por la Secretaría de Ambiente de Bogotá, en el marco del contrato 1467 de 2013 suscrito con la Universidad de La Salle. Los autores agradecen a todas las personas y organizaciones que proporcionaron información o consejos para el desarrollo de este proyecto. 


\section{REFERENCIAS BIBLIOGRÁFICAS}

Agencia Nacional de Infraestructura (ANI) (2013). Informe de Tráfico Por Categoría y estación de los proyectos de concesión carretera a cargo de la ANI y el tráfico Promedio Diario: Tráfico Promedio Diario durante un mes (TPDm) para el período 2012.Bogotá: Agencia Nacional de Infraestructura.

Arciniegas, A.; Rodríguez, C.; Pachón, J.; Sarmiento, H. y Hernández, L. J. (2006a). Estudio de la morbilidad en niños menores a 5 años por enfermedad respiratoria aguda y su relación con la concentración de partículas en una zona industrial de la ciudad de Bogotá. Acta Nova, 3(2), 147-154.

Arciniegas, A.; Rodríguez, C.; Pachón, J.; Sarmiento, H. y Hernández, L.J. (2006b). Relacion entre enfermedad respiratoria aguda en niños menores a cinco años y contaminación atmosférica en Bogotá. Investigaciones en Seguridad Social y Salud, 8, 121-135.

Beltrán, D.; Belalcázar, L.C. y Rojas, N. (2012). Emisiones vehiculares de material particulado (PM2.5 y PM10) por resuspensión de polvo y abrasion de Bogotá. Revista de Ingeniería Sanitaria y Ambiental, 231, 25-33.

Corporación Andina de Fomento (CAF) (2007). Observatorio de Movilidad Urbana: Informe de Movilidad Urbana de Bogotá. Recuperado el 1 de mayo de 2013, de: http://omu.caf.com/media/15663/ omu_ciudad_bogot\%C3\%A1.xls

Cárdenas-Franco, A.H. (2012). Consideraciones del material particulado en Bogotá. Alternativas tecnológicas de medición de la calidad del aire. Tecnura, 13(25), 107-118. Recuperado de: http:// tecnura.udistrital.edu.co/ojs/index.php/revista/ article/view/306

Denby, B.; Horalek, J.; Walker, S.E.; Eben, K. y Fiala, J. (2005). Interpolation and assimilation methods for $\mathrm{Eu}$ ropean scale air quality assessment and mapping Part I: Part I: Review and Recommendations. Bilthoven: European Topic Centre on Air and Climate Change

Franco, J.F.; Rojas, N.; Sarmiento, O.L.; Hernández, L.J.; Zapata, E.; Maldonado, A.; ... Behrentz, E. (2009).
Niveles de material particulado en colegios distritales ubicados en vías con alto tráfico vehicular en la ciudad de Bogotá: estudio piloto. Revista Facultad de Ingeniería Universidad de Antioquia, 49, 101-111.

García U., C.A.; García U., J.C. y Vaca B., M.L. (2012). Emisión de dioxinas y furanos (PCDD/PCDF) en Colombia: evaluación y diagnóstico. Tecnura, 16, 194-206.

Hernández, L.J.; Medina, K.; Cantor, L.; Rodríguez, N.; Barrera, J.; Bayona, J. y Aristizábal, G. (2009). Asociación entre la contaminación del aire y la morbilidad por enfermedad respiratoria aguada en menores de 5 años en tres localidades de Bogotá. Presentación en el II Congreso Colombiano y Conferencia Internacional de Calidad del Aire y Salud Pública, 14 de julio. Cartagena de Indias, Colombia.

Hernández-González, A. y Jiménez, R. (2011). DesarroIlo de un inventario georreferenciado de emisiones de dióxido de carbono por fuentes móviles en el área urbana de Bogotá. Bogotá: Universidad Nacional de Colombia.

Horalek, J.; Denby, B.; DeSmet, P.; DeLeeuw, F.; Brabec, M. y Fiala, J. (2005). Interpolation and assimilation methods for European scale air quality assessment and mapping Part II : Development and testing new methodologies, Bilthoven: European Topic Centre on Air and Climate Change

Ingeniería DICTUC. (2006). Actualización del inventario de emisioines de contaminantes atmosféricos en la región metropolitana de Santiago de Chile 2005. Santiago de Chile: Pontificia Universidad Católica de Chile.

Lents, J.; Walsh, M.; He, K.; Davis, N.; Osses, M.; Tolvett, S. y Hu, L. (2011). Handbook of air quality management. Recuperado de: www.aqbook.org

Ministerio de Minas y Energía (Minminas) (2014). Estadística nacional de conversiones vehiculares a Gas Natural. Recuperado el 1 de mayo de 2014, de: http://www.minminas.gov.co/minminas/downloads/ UserFiles/File/GAS/Gas Natural Vehicular/Conversion/PROGRAMA GNV_DICIEMBRE 2013.pdf 
Organización Mundial para la Salud (OMS) (2006). Guías de calidad del aire de la OMS relativas al material particulado, el ozono, el dióxido de nitrógeno y el dióxido de azufre. Recuperado de: http:// whqlibdoc.who.int/hq/2006/WHO_SDE_PHE_ OEH_06.02_spa.pdf

Organización Mundial para la Salud (OMS) (2014). Air pollution database, by country and city. Recuperado de: www.who.int/quantifying_ehimpacts/national/countryprofile/AAP_PM_database_May2014.xls

Ossés de Eicker, M.; Zah, R.; Triviño, R. y Hurni, H. (2008). Spatial accuracy of a simplified disaggregation method for traffic emissions applied in seven mid-sized Chilean cities. Atmospheric Environment, 42(7), 1491-1502. http://doi.org/10.1016/j. atmosenv.2007.10.079

Pachón, J.E. (2013). Revisión de metodologías usadas para la elaboración de inventarios de emisiones atmosféricas en Colombia: caso de estudio Bogotá. En: Contaminación Atmosférica e Hídrica en Argentina: contribuciones del la IV Reunión Anual PROIMCA y II Reunión Anual PRODECA (pp. 367384). Buenos Aires: Universidad Tecnológica Nacional de Argentina.

Rodríguez, P. y Behrentz, E. (2009). Actualización del inventario de emisiones de fuentes móviles para la ciudad de Bogotá, a través de mediciones directas. Bogotá: Universidad de los Andes.

Rojas, N. y Peñaloza, N. (2010). Distribución espacial y temporal del inventario de emisiones provenientes de las fuentes móviles y fijas de la ciudad de Bogotá. Bogotá: Universidad Nacional de Colombia.

Saide, P.; Zah, R.; Osses, M. y Ossés de Eicker, M. (2009). Spatial disaggregation of traffic emission inventories in large cities using simplified top-down methods. Atmospheric Environment, 43(32), 4914-4923. http://doi.org/10.1016/j.atmosenv.2009.07.013

Secretaría Distrital de Ambiente (SDA) (2011). Plan Decenal de Descontaminación del Aire para Bogotá. Bogotá D.C. Recuperado de: http://ambientebogota.gov.co/en/c/document_library/ get_file?uuid=b5f3e23f-9c5f-40ef-912a-51a5822 da320\&groupld $=55886$
Secretaría Distrital de Ambiente (SDA) (2014). Inventario de emisiones de fuentes móviles con corte a diciembre 2013.Bogotá: Secretaría Distrital de Ambiente.

Secretaría Distrital de Ambiente (SDA) (2015a). Concentración de Material Particulado Inferior a 10 Micrómetros \{PM10\} Promedio Mensual por Estación- PM10PME. Recuperado de: http://oab.ambientebogota.gov.co/es/indicadores?id=876\&v=1

Secretaría Distrital de Ambiente (SDA) (2015b). Número de Motocicletas Particulares- NMtP. Recuperado de: http://oab.ambientebogota.gov.co/es/ indicadores?id=269\&v=1

Secretaría Distrital de Ambiente (SDA) (2015c). Número de Vehículos de Servicio Público Colectivo- NVTPC. Recuperado de: http://oab.ambientebogota. gov.co/es/indicadores?id=270\&v=I

Secretaría Distrital de Ambiente (SDA) y Universidad de Cundinamarca (UDC) (2011). Inventario de emisiones de compuestos orgánicos volátiles (COV) proveniente de fuentes fijas, móviles y de área. Convenio 1360 de 2010. Bogotá: Secretaría Distrital de Ambiente.

Secretaría Distrital de Ambiente (SDA) y Universidad Nacional de Colombia (UNAL) (2013). Informe del Contrato Interadministrativo 013 de 2012. Bogotá: Universidad Nacional de Colombia.

Secretaría Distrital de Movilidad (SDM) y Cal y Mayor y Asociados (C\&M) (2005). Manual de Planeación y Diseño para la Administración del Tránsito y el Transporte. Bogotá: C\&M. 2a edición.

Secretaría Distrital de Movilidad (SDM) (2014). Bases de datos de las estaciones maestras principales para el año 2013, contrato No. 1720 de 2012.

Tuia, D.; Ossés de Eicker, M.; Zah, R.; Osses, M.; Zarate, E. y Clappier, A. (2007). Evaluation of a simplified top-down model for the spatial assessment of hot traffic emissions in mid-sized cities. Atmospheric Environment, 41(17), 3658-3671. http://doi.org/10.1016/j.atmosenv.2006.12.045

Unidad Administrativa Especial de Catastro Distrital (UAECD) (2014). Catálogo de Datos Geográficos, Circular 003 del 24 de enero de 2014. Bogotá: 
Infraestructura de Datos Espaciales para el Distrito Capital (Ideca).

US EPA (1997). Emission Inventory Improvement Program (EIIP). Technical Report Series, volumes I Introduction to the emission inventory improvement program. Morrisville, Carolina del Norte. Recuperado de: http://www.epa.gov/ttnchie1/eiip/techreport/volume01/i01.pdf

US EPA (2004). Developing Spatially Interpolated Surfaces and Estimating Uncertainty, EPA-454/R-04-004.
Morrisville, Carolina del Norte: US Environmental Protection Agency.

Wang, H.; Fu, L.; Lin, X.; Zhou, Y. y Chen, J. (2009). A bottom-up methodology to estimate vehicle emissions for the Beijing urban area. The Science of the Total Environment, 407(6), 1947-53. http://doi.org/10.1016/j.scitotenv.2008.11.008

\section{(9) (1) (8) $\Theta$}

